Results: No significant difference in the mean score was observed between the present study and the normative data. There was a significant effect of tinnitus \& cause of hearing loss on denial of hearing loss subscale and significant association between duration of hearing loss and negative coping strategies subscale. No significant effect of age, gender and degree of hearing loss was noticed on any subscales.

Conclusion: These results suggest that the identification of such factors during routine audiological evaluation can assist the clinician in planning appropriate intervention strategies.

doi:10.1017/S0022215116005594

\section{ID: IP063}

Translation and Cross-cultural Adaptation of Hearing Handicap Inventory for the

EIderly - Screening (HHIE-S) in the

Bilingual Singapore Population - A Pilot

Study

Presenting Author: Eu Chin Ho

Eu Chin Ho, Deborah Lim, Seth Yeak

Tan Tock Seng Hospital

Learning Objectives: Commonly used hearing questionnaires can be translated and cross-culturally adapted into non English languages using a validated translation process.

Introduction: This study aims to to preliminarily assess the validity and reliability of a Mandarin translation of the HHIE-S questionnaire and measure its feasibility.

Method: We adopted a translation process adapted from the MAPI Institute for cross-cultural studies, involving 3 independent forward translations by clinicians, 1 back-translation by a non-clinician and final consensus by a review panel.

15 bilingual patients at Tan Tock Seng Hospital completed the Mandarin HHIE-S after their audiometric assessment. At an interval of about 30 minutes later, patients completed the English HHIE-S. They were given the same Mandarin HHIE-S and instructed to complete one week later.

Results: Mean age of all participants is 70.4 (Standard Deviation 4.42) and mean pure tone audiometry $\left(\right.$ PTA $_{0.5,1,2,4 \mathrm{kHz}}$ ) of the better ear was $34.58 \mathrm{~dB}$ (Standard Deviation 14.34). Self-rated English and Mandarin language proficiencies were comparable.

\section{Validity of Mandarin HHIE-S}

1. Mandarin and English total scores are significantly correlated, showing a strong positive relationship (Pearson Coefficient $0.885, \mathrm{p}<0.001$ ).

2. Analysis of HHIE-S categories of non-significant hearing-loss and Significant hearing-loss with corresponding categories on Gold Standard criterion of PTA $_{0.5,1,2,3 \mathrm{kHz}}$ showed poor agreement (Kappa coefficient $=0.0367,<0.2$ ). User feedback was sought regarding problematic questions.

\section{Reliability}

1. Internal consistency of the 10 items in the Mandarin HHIE-S is good with a Cronbach's alpha of 0.8499 $(>0.8)$.

2. Test-retest reproducibility of the first and second Mandarin HHIE-S at one-week interval is strong. Among $80 \%$ of patients, difference between the 2 mandarin scores is less than 5 .

User acceptance scores were positive in terms of readability, comprehensibility and relevance.

Conclusion: The Mandarin HHIE-S demonstrated high reliability though low validity. A larger scale validation study incorporating user feedback from this pilot study should be carried out in the future.

doi:10.1017/S0022215116005600

\section{ID: IP064}

Utility of WHODAS 2.0 (Quality of Life Assessment) in detecting Changes in Quality of Life in Hearing Impairment

Presenting Author: Eu Chin Ho Eu Chin Ho

\section{Tan Tock Seng Hospital}

Learning Objectives: WHODAS 2.0 may not be sensitive enough in detecting quality of life change in hearingrelated disability.

Introduction: The objective of this study is to evaluate the relationship of pure tone audiogram (PTA) score, screening questionnaire and quality of life assessment via World Health Organisation Disability Assessment Schedule 2.0(WHODAS 2.0) in our Singaporean population.

Methods: A retrospective review of 56 participants who were recruited for hearing screening held in a Singaporean Tertiary General Hospital from 29-30th May 2013 was done. Information recorded include general demographics, self-perception of hearing level, Hearing Handicap Inventory for Elderly Screening (HHIE-S) for participants $>60$ years old, Hearing Handicap Inventory for Adults (HHIA) for participants PTA $(0.5,1,2,4 \mathrm{kHz})$ done by an experienced audiologist. WHODAS 2.0 was measured as a total global score (maximum: 100) as well as specific domain scores for 6 domains: Cognition, Mobility, SelfCare, Getting along, Life activities and Participation.

Results: The mean total WHODAS score was 42.3 (Range: 32-96, SD: 14.5). Mean specific domain scores include: Cognition at 8.9 (Range: 6-19, SD: 3.6), Mobility at 6.6 (Range: 5-17, SD: 3.02), Self-Care at 4.4 (Range: 5-12, SD: 1.53), Getting along at 6.1 (Range: 5-17, SD: 2.49), Life activities at 5.14 (Range: 4-14, SD: 2.54).

There was no significant correlation found between total WHODAS score and PTA score of better ear $(p=0.322)$ and between domain specific WHODAS score and PTA score, except for the Getting Along domain $(\mathrm{r}=0.26, \mathrm{p}=0.031)$. There was also no significant correlation found between HHIA or HHIE and any WHODAS score (all p $>0.05$ ). 